\title{
Protocol Synthesis for Real-Time Applications
}

\author{
AHMED KHOUMSI \\ Department GEGI, University of Sherbrooke, Sherbrooke, Canada \\ GREGOR V. BOCHMANN \\ School of ITO, University of Ottawa, Ottawa, Canada \\ RACHIDA DSSOULI \\ Department IRO, University of Montreal, Montreal, Canada
}

Key words: Real-Time Protocol, Service, Synthesis, Assembly System, X.25 Protocol.

\begin{abstract}
This paper deals with deriving protocol specifications which provide a given service satisfying timing requirements. In previous work, we have developed an extension of a method proposed by Saleh and Probert, by considering timing requirements in a more general case than in other existing studies. In the present paper, we improve our method by several modifications and additions. First, the number of messages exchanged between the protocol entities is minimized. Second, a less restrictive strategy for choosing between several service primitives is proposed. Third, we consider applications where the choice between several primitives of a single site can be made by the user, and not only by the system. Fourth, conditions of existence of solutions are weaker. Fifth, the timing constraints of the synthesized protocols are weaker. Finally, two simple examples of applications are described.
\end{abstract}

\section{INTRODUCTION}

Several methods for deriving a protocol from a service have been developed by various researchers $[3,5-7,10,11]$. These methods are not applicable for real-time applications, for which the correct ordering of service primitives alone does not always ensure the success of a task. In addition, certain delays must be respected between occurrences of services primitives. In [6] transit delays in the medium are supposed negligible, while in [7] they are bounded by a maximum value. In the present paper, our aim is to describe an approach for deriving protocols which guarantee both : (a) a correct ordering of service primitives; and (b) the satisfaction of given timing requirements between the executions of service primitives, in a more general case than in [6,7].

A protocol derivation approach presented in [10], which guarantees only (a), has been extended in [8] to the real-time case, i.e., for ensuring also (b). The timing requirements considered in [8] allow to specify certain constraints on the delays between consecutive service primitives. In the present paper, we consider the same problem but we provide a better 
solution. In fact, compared to [8] : (a) the number of messages exchanged between protocol entities for providing a desired service has been minimized; (b) the strategy for choosing between several possible service primitives is distributed among several sites, instead of being centralized in a single site; (c) we consider applications where the choice between several primitives of a single site can be made by the user, and not only by the system; (d) conditions of existence of solutions are weaker; (e) the temporal constraints to be respected by the protocol entities are weaker. We have also presented two concrete examples of application.

The remaining of this paper is organized as follows. In Sect. 2, we introduce the problem of protocol derivation and the principle used for deriving protocols. Sect. 3 deals with non-real-time systems. First, we show how services and protocols are specified, and then we introduce the method for deriving protocols. Sect. 4 to 7 deal with real-time systems. In Sect. 4, we describe how services and protocols are specified. In Sect. 5, we explain the approach used for calculating temporal requirements for protocol entities from temporal requirements of a service to be provided. We also present some rules for deriving real-time protocols in a systematic way. In Sect. 6 and 7, two examples illustrate our method. Finally, we conclude in Sect. 8.

\section{PROTOCOL SYNTHESIS}

We consider a distributed system (DS) consisting of several sites interconnected through a reliable communication medium (simply called medium). We assume that: (a) each pair of sites can communicate with each other through the medium; (b) the environment can interact with the DS at the different sites through service access points (SAP). These interactions correspond to the executions of service primitives (simply called primitives). We may assume that to each $\mathrm{Sit}_{\mathrm{i}}$ corresponds a protocol entity $\mathrm{PE}_{\mathbf{i}}$.

In the user's viewpoint, the DS provides a service where only executions of primitives are visible. We assume that the specification of the desired service (provided to the user) defines the ordering of and the timing requirements between the occurrences of primitives. The timing requirements define the real-time properties of the DS.

The problem of the designer is then : How can he derive systematically specifications of the local $\mathrm{PE}_{\mathrm{i}}$, for $\mathrm{i}=1,2, \ldots, \mathrm{n}$, from the specification of the desired service. In the case of a real-time system, the designer must also have a temporal model of the medium, which is assumed reliable.

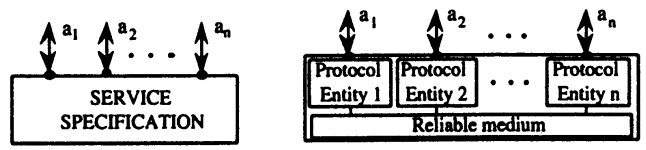

Figure 1. Service and protocol concepts

The approach we have selected for deriving protocols is called Synthesis, and the systems considered are assumed sequential. The basic principle we have used is the following: if in the specification of the service, a primitive 
$A$ is executed by $\mathrm{PE}_{\mathrm{a}}$, and is followed by the execution of a primitive $\mathrm{B}$ by $P E_{b}$, then: $(\alpha)$ after $P E_{a}$ executes $A$, it sends a message $m$ to $P E_{b} ;(\beta)$ after $P E_{b}$ receives $m$, it executes $B$.

\section{PROTOCOL SYNTHESIS FOR NON-REAL- TIME APPLICATIONS}

Service specification : A service desired by the user is described by a finite state automaton $(\mathcal{F S A})$, denoted SS, which specifies the sequences of primitives the user would like to observe at the various SAPS. Every transition of SS (Fig. 2) is defined by [q, $\left.E_{a}, r\right]$, where: (1) $q$ and $r$ are origin and destination states; and (2) $\mathrm{E}_{\mathrm{a}}$ represents a primitive $\mathrm{E}$ executed by $\mathrm{PE}_{\mathrm{a}}$. Besides, every transition is identified by a number $p$ and then denoted $\mathrm{T}_{\mathrm{p}}=\left[\mathrm{q}, \mathrm{E}_{\mathrm{a}}, \mathrm{r}\right]$. In a figure representing an $\mathcal{F S A}$, every transition $\mathrm{T}_{\mathrm{p}}=\left[\mathrm{q}, \mathrm{E}_{\mathrm{a}}, \mathrm{r}\right]$ may be simply represented by $T_{p}=E_{a}$ when $q$ and $r$ are explicitly represented by the transition diagram.

Definition 3.1. (Incoming and outgoing transitions).

An outgoing (resp. incoming) transition of a state $\mathrm{q}$ is a transition which is executable from (resp. leads to) $\mathrm{q}$.

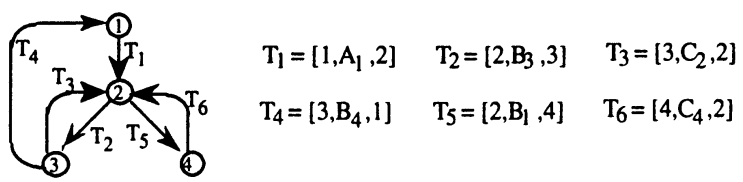

Figure 2. Service specification

Protocol specification : A PE $\mathrm{a}$ is described by an $\mathcal{F S A}$, denoted $\mathrm{PS}_{\mathrm{a}}$ (see for example Fig. 5), which specifies the sequences of events occurring at Site $\mathrm{a}_{\mathrm{a}}$. There are three types of events in each $\mathrm{PS}_{\mathrm{a}}$.

Type $P$ (for Primitive): The execution of a primitive $\mathrm{E}$ is denoted $\mathrm{E}_{\mathrm{a}}$.

Type $S$ (for Send): The sending of a message is denoted $\mathrm{s}_{\mathrm{a}}^{\mathrm{i}}(\mathrm{p})$, and means "message containing by $\mathrm{p}$ is sent by $\mathrm{PE}_{\mathrm{a}}$ to entity $\mathrm{PE}_{\mathrm{i}}$ ".

Type $R$ (for Receive): The reception of a message is denoted $\mathrm{r}_{\mathrm{a}}^{\mathrm{i}}(\mathrm{p})$, and means "message containing $\mathrm{p}$ and coming from $\mathrm{PE}_{\mathrm{i}}$ is received by $\mathrm{PE}_{\mathrm{a}}$ ".

Let $\mathrm{PE}_{1}, \mathrm{PE}_{2}, \ldots, \mathrm{PE}_{\mathrm{n}}$ be specified by $\mathrm{PS}_{1}, \mathrm{PS}_{2}, \ldots, \mathrm{PS}_{\mathrm{n}}$ respectively. Let $\mathcal{D S}$ be constituted by $\mathrm{PE}_{1}, \mathrm{PE}_{2}, \ldots, \mathrm{PE}_{\mathrm{n}}$ and by the medium, and specified by $\mathrm{DS}$.

Definition 3.2. (Combined behaviour of several PES) The combined behaviour of $\mathrm{PE}_{1}, \ldots, \mathrm{PE}_{\mathrm{n}}$ is the behaviour of $\mathcal{D S}$. The specification DS of this behaviour can be computed from $\mathrm{PS}_{1}, \ldots, \mathrm{PS}_{\mathrm{n}}$. We define this combined behaviour by the operator $C o m b: \mathrm{DS}=\operatorname{Comb}\left(\mathrm{PS}_{1}, \mathrm{PS}_{2}, \ldots, \mathrm{PS}_{\mathrm{n}}\right)$.

Definition 3.3. (Projection, total and partial provision of a desired service) Let $V_{S}$ and $V_{i}$ be the alphabets of the service and $P S_{i}$, respectively. We also 
use the following concepts : (i) $\operatorname{Proj} \Lambda$ (A) denotes the projection of an $\mathcal{F S A} A$ into an alphabet $\Lambda$; as an example, Projv $\mathrm{V}_{\mathbf{S}}(\mathrm{DS})$ specifies the service provided to the user by $\mathcal{D S}$; (ii) $\mathrm{A} \cong \mathrm{B}$ means that the $\mathcal{F S}$ As $\mathrm{A}$ and $\mathrm{B}$ accept the same language; (iii) $A<B$ means that the language accepted by $A$ is included in the one accepted by B. We say that the service is totally (resp. partially) provided if $\operatorname{Proj}_{\mathbf{s}}(\mathrm{DS}) \cong S S$ (resp. $\left.\operatorname{Proj}_{\mathbf{s}}(\mathrm{DS})<\mathrm{SS}\right)$.

Definition 3.4. (Semantic and syntactic correctness)

We say that the protocol is semantically correct if the desired service is totally provided. The protocol is syntactically correct if DS is deadlock-free and livelock-free and no unspecified reception error is possible (we assume that the desired service SS is deadlock-free and livelock-free).

Our aim is therefore to propose a synthesis method which, from the specification of a service, generates specifications of protocol entities which are syntactically and semantically correct.

Principle for deriving PEs : From an SS specifying a desired service, deriving a protocol consists of generating an $\mathcal{F S A} \mathrm{PS}_{\mathrm{i}}$ for each $\mathrm{Site}_{\mathrm{i}}$, which specifies the action sequences executed at $\mathrm{Site}_{\mathrm{i}}$. In order to provide the service, the different $P$ ES exchange messages through a medium. The basic principle used for deriving a protocol (see Sect. 2) has been applied in [8] as follows. If the execution of a primitive $A$ by $\mathrm{PE}_{\mathrm{a}}$ is followed by a choice between primitives executed by other $P E_{b i} s$, for $i=1, \ldots, k$, (Fig. 3 ) then, after the execution of $\mathrm{A}, \mathrm{PE}_{\mathrm{a}}$ decides which transition should follow. It therefore sends a message $m$ to all $P E_{b i} s(i=1, \ldots, k$, and $b i \neq a)$ which contains two parameters: $p$ which identifies the executed transition $\mathrm{T}_{\mathrm{p}}$; and $q$ which identifies the chosen transition $T_{q}$ to be executed next. All $\mathrm{PE}_{\mathrm{bi}} \mathrm{s}$ will receive $\mathrm{m}$, but only one of them will execute the primitive corresponding to the selected transition $\mathrm{T}_{\mathrm{q}}$.

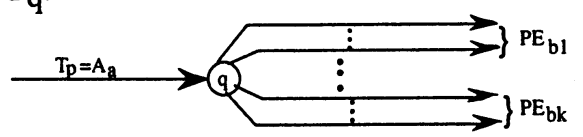

Figure 3. Choice between several actions

This principle implies that the following three restrictions must be satisfied.

Restriction 1. The transitions which may occur in the initial state of SS are all executable by $a$ single $P E$.

Restriction 2. The choice between several primitives is made by the system and not by the user.

Restriction 3. The choice between primitives executed by a given $\mathrm{PE}_{\mathrm{bi}}$ is made by $\mathrm{PE}_{\mathrm{a}}$.

Compared to $[8,10]$, the following three improvements are made in the subsections below: 
(a) Restriction 2 is weakened as follows: the choice between $\mathrm{PE}_{\mathrm{bi}} \mathrm{s}$ is made at Site $_{\mathrm{a}}$ by the system, but the choice between several primitives of the selected $\mathrm{PE}_{\mathrm{bi}}$ may be made at the selected site by the system (for upward primitives) or by the user (for downward primitives).

(b) Restriction 3 is removed as follows: $\mathrm{PE}_{\mathrm{a}}$ is not necessarily required to select the following primitive; it may decide to select only the following PE which, in turn, selects one of its primitives.

(c) $\mathrm{PE}_{\mathrm{a}}$ sends a message only to the selected PE.

Derivation procedure : SS being the input of the problem, the derivation procedure consists of the following two steps.

Step 1 : This step consists of completing SS by the insertion of a message exchange between each pair of consecutive primitives which are executed in different sites. In order to avoid any ambiguity, every message contains the number of the transition in SS which is associated to the first of the two primitives. The obtained specification is denoted GPS.

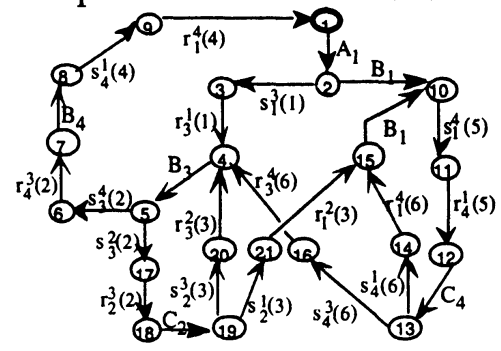

Figure 4. GPS obtained in Step 2 for the example represented in Fig. 2

Step 2 : From GPS, the specification $\mathrm{PS}_{\mathrm{i}}$ of each $\mathrm{PE}_{\mathrm{i}}$ is obtained by projecting GPS into the alphabet of events which occur in Site $e_{i}$. Then, the $\mathrm{PS}_{\mathrm{i}}$ obtained are minimized and determinized.

We note that this two-step procedure is simpler than the procedures proposed in $[8,10]$, besides being optimal. For our example of Fig. 2, we obtain the specifications of Figures 4 and 5, after the first and second steps, respectively. To make the projections of GPS (Fig. 4) for obtaining PS to 4) (Fig. 5) more directly visible, the states of $P_{i}$ are named according to their corresponding states in GPS, where " $\mathrm{i}-\mathrm{j}$ " means all integers from $\mathrm{i}$ to $\mathrm{j}$.

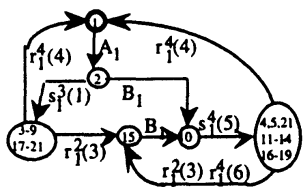

(a) $\mathrm{PS}_{1}$

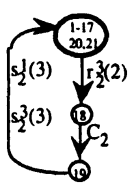

(b) $\mathrm{PS}_{2}$

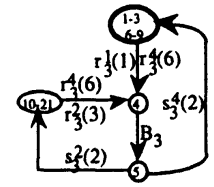

(c) $\mathrm{PS}_{3}$

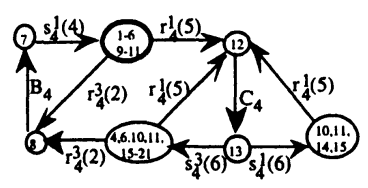

(d) $\mathrm{PS}_{4}$

Figure 5. Obtained protocol specifications

Contrary to [10], the rules for deriving PEs do not depend on whether primitives are upward or downward. In our opinion, such a distinction 
complicates uselessly the rules of Step 1. In the remaining part of this paper, we extend the procedure of protocol derivation to real-time systems.

\section{TIMED AUTOMATA TO SPECIFY SERVICES AND PROTOCOLS}

We use a dense-time model $[1,8]$ where the time is viewed as a state variable that ranges over a dense domain and evolves indefinitely. The timed automata $(\mathcal{T A})$ model we propose here uses a variable $\mathbf{v}$ and a clock $\mathbf{c}$.

Definition 4.1. (Clock c, variable v)

c has a positive real value which : (1) is set to zero at the occurrence of every transition; and (2) is equal to the time elapsed since the last instant it was set to zero. $v$ has a strictly positive natural value which can be updated at the occurrence of any transition.

Let $\mathrm{A}=\left(\mathrm{Q}, \Sigma, \delta, \mathrm{q}_{0}\right)$ be an $\mathcal{F S A}$ where $\mathrm{Q}$ is a set of states, $\Sigma$ is an alphabet, $\mathrm{q}_{0}$ is the initial state, and $\delta \subseteq \mathrm{Q} \times \Sigma \times \mathrm{Q}$ defines the transitions. Let us see how a $\mathcal{T A}$ can be defined from the $\mathcal{F S A}$ A.

Definition 4.2. (Timed transition, and Timed automaton) Let $\mathrm{I}=[\mathrm{a} ; \mathrm{b}]$ be an interval, where $\mathrm{a}$ and $\mathrm{b}$ are positive real numbers and $\mathrm{a} \leq \mathrm{b}$. A timed transition is defined by $[\mathrm{q}, \sigma, \mathrm{r} ; \boldsymbol{C}, v]$ where : (a) $[\mathrm{q}, \sigma, \mathrm{r}]$ defines a transition of the $\mathcal{F S A} \mathcal{A} ;$ (b) $C=\left(\mathrm{I}_{1}, \mathrm{I}_{2}, \ldots, \mathrm{I}_{m}\right)$ is a $m$-tuple of non-empty intervals; and (c) $v$ is a value of variable $v$. A $\mathcal{T A} \mathrm{A}^{\mathrm{t}}$ can therefore be constructed if we transform every transition $\operatorname{tr}=\left[q_{1}, \sigma, q_{2}\right]$ of $A$ into a timed transition $\operatorname{Tr}$ by associating to it an $m$-tuple $C$ of intervals and a value $v$ of $\mathbf{v}$. The semantics of a timed transition $\operatorname{Tr}=[\mathrm{q}, \sigma, \mathrm{r} ; \boldsymbol{C}, v]$ of $\mathrm{A}^{\mathrm{t}}$ depends on the current state $\mathrm{q}$ and on the current values of $\mathrm{v}$ and $\mathrm{c}$ as follows. If $u$ is the current value of $v$ then : (1) $\mathrm{Tr}$ is enabled (i.e., may occur) only if the current value of $c$ falls within the $u$ th interval $I_{u}$ of $C$; and (2) after the occurrence of $\operatorname{Tr}, \mathbf{v}$ is set to $v$ and $\mathbf{c}$ is set to zero. Intuitively, the temporal constraint of a transition may depend on how the current state has been reached (this information is given by $\mathbf{v}$ ).

Henceforth, every timed transition is simply called transition, and $\operatorname{Tr}=[q, \sigma, r ; \mathcal{C}, v]$ may be simply represented by $\operatorname{Tr}=[\sigma ; \mathcal{C}, v]$ if there is no ambiguity about $q$ and $r$. An example of a part of $\mathcal{T A}$ is given in Fig. 6. State $q$ has two incoming $\left(\operatorname{Tr}_{1}\right.$ and $\left.\operatorname{Tr}_{2}\right)$ and two outgoing transitions ( $\operatorname{Tr}_{3}$ and $\left.\operatorname{Tr}_{4}\right)$, with $\operatorname{Tr} 1=[\mathrm{q} 1, \sigma 1, \mathrm{q} ; C 1, v 1], \operatorname{Tr}_{2}=[\mathrm{q} 2, \sigma 2, \mathrm{q} ; C 2, v 2], \operatorname{Tr} 3=[\mathrm{q}, \sigma 3, \mathrm{r} 1 ; C 3, v 3]$, and $\operatorname{Tr}_{4}=\left[\mathrm{q}, \sigma 4, \mathrm{r} 2 ; C_{4}, v^{4}\right]$. With the representation of Fig. 6, we can define $v 1$ and $v 2$, and $C 3$ and $C 4$. In fact, for a timed transition $\operatorname{Tr}=[q, \sigma 1, \mathrm{r} ; C, v]$, the definition of $v$ necessitates to know all the incoming transitions of $r$, and the definition of $C$ necessitates to know all the incoming transitions of q. For example, $\quad v 1=1, \quad v 2=2, \quad C 3=\left(\mathrm{I}_{1}, \mathrm{I} 3_{2}\right), \quad C 4=\left(\mathrm{I} 4_{1}, \mathrm{I} 4_{2}\right), \quad \mathrm{I} 3_{1}=[1 ; 2], \quad \mathrm{I} 3_{2}=[0 ; 2]$, 
$\mathrm{I} 4_{1}=[1 ; 3]$ and $\mathrm{I}_{2}=[2 ; 5]$. The informal specification is then the following, with $q$ being the current state:

- if $v=1$, i.e., $q$ has been reached by transition $\operatorname{Tr}_{1}$, then :

* $\operatorname{Tr}_{3}$ (resp. Tr 4 ) may occur after a delay within the interval I3 ${ }_{1}$ (resp. I4 1 );

* If neither $\operatorname{Tr}_{3}$ nor $\operatorname{Tr}_{4}$ occurs after a delay within [1;2], then $\operatorname{Tr}_{4}$ must occur after a delay within [2;3] (in order to avoid a deadlock).

- if $v=2$, i.e., $q$ has been reached by the transition $\operatorname{Tr}_{2}$, then :

* $\operatorname{Tr}_{3}$ (resp. $\operatorname{Tr}_{4}$ ) may occur after a delay within the interval $\mathrm{I}_{2}$ (resp. I42);

* If $\operatorname{Tr} 3$ does not occur after a delay within [0;2], then $\operatorname{Tr}_{4}$ must occur after a delay within $[2 ; 5]$.

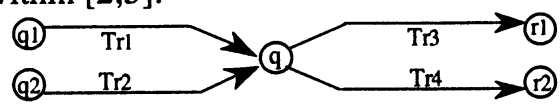

Figure 6. Incoming and outgoing transitions

A desired service is described by a $\mathcal{T A}$ denoted SST, which specifies: (a) the required sequences of primitives; and (b) certain temporal requirements between consecutive primitives. In any state q of SST, we can express some temporal constraints on the primitives which are executable from state q. These temporal constraints may depend on how $\mathrm{q}$ has been reached. As an example, the $\mathcal{F S A}$ SS of Fig. 2 (Sect. 3) is transformed into a $\mathcal{T A}$ SST by replacing transitions $T_{i}$ of $S S$ into the following timed transitions $\operatorname{Tr}_{i}$, $\mathrm{i}=1, \ldots, 6, \quad$ respectively : $\operatorname{Tr}_{1}=\left[1, \mathrm{~A}_{1}, 2 ; C 1,1\right], \quad \operatorname{Tr}_{2}=\left[2, \mathrm{~B}_{3}, 3 ; C 2,1\right]$, $\operatorname{Tr}_{3}=\left[3, \mathrm{C}_{2}, 2 ; C 3,2\right], \operatorname{Tr}_{4}=\left[3, \mathrm{~B}_{4}, 1 ; C_{4}, 1\right], \operatorname{Tr}_{5}=\left[2, \mathrm{~B}_{1}, 4 ; C_{5}, 1\right], \operatorname{Tr}_{6}=\left[4, \mathrm{C}_{4}, 2 ; C 6,3\right]$, and $C_{1}=\mathrm{I} 1, C 2=\left(\mathrm{I} 2_{1}, \mathrm{I} 2_{2}, \mathrm{I} 2_{3}\right), C_{3}=\mathrm{I} 3, C 4=\mathrm{I} 4, C_{5}=\left(\mathrm{I}_{1}, \mathrm{I} 5_{2}, \mathrm{I} 5_{3}\right), C_{6}=\mathrm{I} 6$, where $\mathrm{I} 1$, $\mathrm{I} 2_{1}, \mathrm{I} 2_{2}, \mathrm{I} 2_{3}, \mathrm{I} 3, \mathrm{I} 4, \mathrm{I} 5_{1}, \mathrm{I} 5_{2}, \mathrm{I} 5_{3}$, and $\mathrm{I} 6$ are intervals. For example, if the current state 2 is reached by $\operatorname{Tr} 3$, then $\mathbf{v}$ is set to $2, \operatorname{Tr}_{2}$ is enabled iff ( $c \in I_{2}$ ) and $\operatorname{Tr}_{5}$ is enabled iff $\left(c \in I 5_{2}\right)$ (intervals $I 2_{2}$ and $I 5_{2}$ of $C 2$ and $C 5$ are used because $v=2$ ). Therefore, if "I $\leftarrow\left\langle\operatorname{Tr}_{\mathrm{a}}, \operatorname{Tr}_{\mathrm{b}}\right\rangle$ " means "the delay of $\operatorname{Tr}_{\mathrm{a}}$ and $\operatorname{Tr}_{\mathrm{b}}$ falls within the interval I" then : I1 $\leftarrow\left\langle\operatorname{Tr}_{4}, \operatorname{Tr}_{1}\right\rangle \quad \mathrm{I}_{1} \leftarrow\left\langle\operatorname{Tr}_{1}, \operatorname{Tr}_{2}\right\rangle$ $\mathrm{I}_{2} \leftarrow\left\langle\operatorname{Tr}_{3}, \operatorname{Tr}_{2}\right\rangle \quad \mathrm{I}_{3} \leftarrow\left\langle\operatorname{Tr}_{6}, \operatorname{Tr}_{2}\right\rangle \quad \mathrm{I} 3 \leftarrow\left\langle\operatorname{Tr}_{2}, \operatorname{Tr}_{3}\right\rangle \quad \mathrm{I} 4 \leftarrow\left\langle\operatorname{Tr}_{2}, \operatorname{Tr}_{4}\right\rangle$ $\mathrm{I}_{1} \leftarrow\left\langle\operatorname{Tr}_{1}, \operatorname{Tr}_{5}\right\rangle \quad \mathrm{I}_{2} \leftarrow\left\langle\operatorname{Tr}_{3}, \operatorname{Tr}_{5}\right\rangle \quad \mathrm{I}_{3} \leftarrow\left\langle\operatorname{Tr}_{6}, \operatorname{Tr}_{5}\right\rangle \quad \mathrm{I} 6 \leftarrow\left\langle\operatorname{Tr}_{5}, \operatorname{Tr}_{6}\right\rangle$.

A $\mathrm{PE}_{\mathrm{a}}$ is described by a $\mathcal{T A}$ denoted PST $\mathrm{P}_{\mathrm{a}}$, which specifies: (a) the sequences of events which occur at $\mathrm{Site}_{\mathrm{a}}$; (b) certain temporal constraints to be satisfied between consecutive events. Similarly to the non-real-time case, the events may be of the three types $P, S$ and $R$. Examples of $\mathcal{T A s}$ specifying PEs are given in Sect. 6 and 7.

We consider $\mathrm{PE}_{1}, \mathrm{PE}_{2}, \ldots, \mathrm{PE}_{\mathrm{n}}$ which are specified by $\mathrm{PST}_{1}, \mathrm{PST}_{2}, \ldots$, $\mathrm{PST}_{\mathrm{n}}$, respectively. Let $\mathcal{D S}$ be constituted by $\mathrm{PE}_{1}, \mathrm{PE}_{2}, \ldots, \mathrm{PE}_{\mathrm{n}}$ and by the medium, and specified by a $\mathcal{T A D S T}$.

Definition 4.3. (Timed sequence of events, Timed language, Acceptance) 
A timed sequence $\mathrm{T}$ is represented by $\left\langle\sigma_{1}, t_{1}\right\rangle\left\langle\sigma_{2}, t_{2}\right\rangle \ldots\left\langle\sigma_{i}, t_{1}\right\rangle \ldots$ and means that events $\sigma_{1}, \sigma_{2}, \ldots, \sigma_{\mathrm{i}}, \ldots$ occur at instants $t_{1}, t_{2}, \ldots, t_{1} \ldots$, respectively, where 0 $<t_{1}<t_{2}<\ldots<t_{1}<\ldots$ A timed language is a set (possibly infinite) of timed sequences. Let $\mathrm{A}$ be a $\mathcal{T A}$, and $\mathrm{L}_{\mathrm{A}}$ be the set of sequences which can be executed by $A$. Then we say that $A$ accepts the language $L_{A}$.

Definition 4.4. (Projection, total and partial provision of a desired service with temporal requirements) The projection of a $\mathcal{T A}$ into a subalphabet $\Lambda$ can be defined similarly to the projection of an $\mathcal{F S A}$. Therefore, ProjV $\mathrm{V}_{\mathbf{s}}(\mathrm{DST})$ specifies the service provided to the user by $\mathcal{D S}$, and each $\operatorname{Projv}_{\mathrm{i}}(\mathrm{DST})$ specifies $P E_{i}$, for $i=1, \ldots, n$. For the comparison of timed languages, we use the symbols $\cong \mathrm{T}$ and $<_{\mathrm{T}}$, i.e., $\mathrm{A} \cong \mathrm{T}$ means that $\mathrm{L}_{\mathrm{A}}=\mathrm{L}_{\mathrm{B}}$, and $\mathrm{A}<_{\mathrm{T}} \mathrm{B}$ means that $L_{A} \subset L_{B}$. Total and partial provisions of a real-time service are defined like in Def. 3.3, but by using symbols $\cong \mathrm{T}$ and $<\mathrm{T}$ instead of $\cong$ and $<$.

\section{PROTOCOL SYNTHESIS FOR REAL-TIME APPLICATIONS}

Definition 5.1. (Reliable medium) Besides not altering messages, in the realtime case a reliable communication medium must be such that the transit delay $t_{m}$ of a message sent at Site $_{a}$ and received at Site $e_{b}$, belongs to a finite interval $M_{a, b}=\left[\mu_{a, b} ; \rho_{a, b}\right]$ which depends on Site $e_{a}$ and Site S.

The synthesis of the real-time PEs uses the same Step 1 of the non-realtime case, where we obtain GPST from SST (the last $T$ indicates the presence of temporal constraints). In this step, the timed transitions are processed like simple transitions, while $C$ and $v$ are kept unchanged. GPST specifies the correct ordering of primitives, but it does not specify the correct temporal requirements of the service.

To compute temporal constraints for the PEs, we consider every pair of states $q$ and $r$ of GPST which are connected by two consecutive events $s_{a}^{b}(p)$ and $r_{b}^{a}(p)$ (see Fig. 7). Let $T r$ be the single incoming transition of $q$ (which corresponds to a primitive executed at a Site $a$ ) and let $\operatorname{Tr}_{1}, \ldots, \operatorname{Tr}_{n}$ be the outgoing transitions of $\mathbf{r}$ (which correspond to primitives executed at the same $S_{i t e}$ ). After $T r, P E_{a}$ sends a message to $P_{b}$ (written $s_{a}^{b}(p)$ ); when $P E_{b}$ receives the message (written $r_{b}^{a}(p)$ ), it executes one of the $n \operatorname{Tr}_{k}$. The sequencing of events between $\operatorname{Tr}=[\mathrm{q} 1, \sigma, \mathrm{q} ; C, v]$ and $\operatorname{Tr}_{\mathrm{k}}=\left[\mathrm{r}, \sigma \mathrm{k}, \mathrm{q} 2 ; \mathcal{C}_{\mathrm{k}}, v_{\mathrm{k}}\right]$ is represented as a function of the time in Fig. 8.a. The delay between $\operatorname{Tr}$ and $\operatorname{Tr}_{\mathrm{K}}$ must belong to the $\imath$ th interval $\mathrm{Ik}_{v}=\left[\gamma \mathrm{k}_{v} ; \delta \mathrm{k}_{v}\right]$ of $C$ which, for simplicity, is denoted $\mathrm{I}_{\mathrm{k}}=\left[\gamma_{\mathrm{k}} ; \delta_{\mathrm{k}}\right]$.

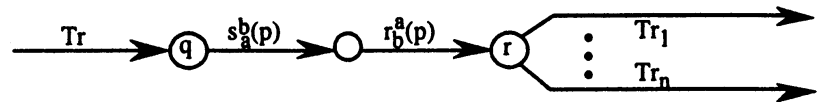

Figure 7. Outgoing transitions on a state of GPST 


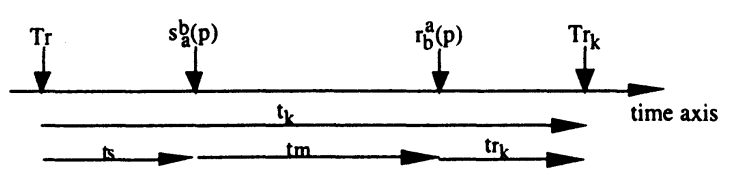

(a) Representation in function of time

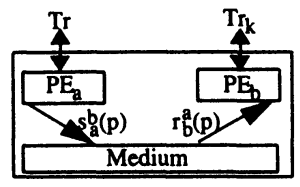

(b) Representation by entities

Figure 8. Representation of events between $\operatorname{Tr}$ and $\operatorname{Tr}_{k}$

From Fig. 8.a, we see that the service requires that the time $t_{k}$, between the executions of $\operatorname{Tr}$ and $\operatorname{Tr}_{k}$, falls within $\mathrm{I}_{k}=\left[\gamma_{\mathrm{k}} ; \delta_{\mathrm{k}}\right]$. The model of the medium implies that the transit delay tm of a message sent by $\mathrm{PE}_{\mathrm{a}}$ and received by $P E_{b}$, falls within $M_{a, b}=\left[\mu_{a, b} ; \rho_{a, b}\right]$. The aim of the temporal requirements derivation for the protocol entities is the following.

From requirements $t m \in M_{a, b}=\left[\mu_{a, b} ; \rho_{a, b}\right]$ and $t_{k} \in I_{k}=\left[\gamma_{k} ; \delta_{k}\right](k=1,2, \ldots, n)$, we must compute constraints on ts and $\operatorname{tr}_{k}(k=1,2, \ldots, n)$ which ensure that requirements $t_{k} \in I_{k}$ on the service will be respected. These derived constraints are written in the form $\mathrm{ts} \in \mathrm{S}=[\theta ; \phi]$, and $\operatorname{tr}_{\mathrm{k}} \in \mathrm{R}_{\mathrm{k}}=\left[\tau_{\mathrm{k}} ; \omega_{\mathrm{k}}\right], \mathrm{k}=1$, $2, \ldots, \mathrm{n}$. This computation must be made for each occurrence of the structure in Fig. 7 within GPST.

Notations: operators $ᄃ, \cup$ or $\cap$ will be used on intervals, and $[a ; b]+[c ; d]=[a+c ; b+d],[a ; b]-[c ; d]=[a-c ; b-d]$.

Condition for existence of solutions : we consider two consecutive transitions $\operatorname{Tr}_{1}$ and $\operatorname{Tr}_{2}$ wich are executed at $\mathrm{Site}_{\mathrm{i}}$ and $\mathrm{Site}_{\mathrm{j}}$, respectively. After $\operatorname{Tr}_{1}$, Site $e_{i}$ sends a message to Site $j_{j}$ to inform it that it may execute $\operatorname{Tr}_{2}$. If the delay between $\operatorname{Tr}_{1}$ and $\operatorname{Tr}_{2}$ must be greater than $x$ and smaller than $y$, then the transit delay of the message must be smaller than y. Besides, the difference between the biggest delay and the smallest delay of the message in the medium must be smaller than $y$-x. Formally, for each occurrence of the structure in Fig. 7 within the GPST, we must have :

$$
\text { for } \mathrm{k}=1,2, \ldots, \mathrm{n}: \quad \delta_{\mathrm{k}}-\rho_{\mathrm{a}, \mathrm{b}} \geq \sup \left(\gamma_{\mathrm{k}}-\mu_{\mathrm{a}, \mathrm{b}} ; 0\right)
$$

where $\sup (a ; b)$ is equal to the biggest of $a$ and $b$.

Therefore, for each occurrence within GPST of the structure in Fig. 7, we must check if (1) is respected. If the checking is positive then we must compute : (a) the interval $S$ representing the constraint on ts, and (b) intervals $R_{k}, k=1,2, \ldots, n$, representing the constraints on $\operatorname{tr}_{k}, k=1,2, \ldots, n$.

We note that condition (1) is less restrictive than the conditions for the existence of solutions of [8]. For resolving the timing constraints of the PES, we consider the following three cases :

Static case : messages transmitted by PES contain no temporal information; First dynamic case : the PES include some temporal information in the messages they send;

Second dynamic case : the temporal information included by the PES is completed by the medium. 
In the following, $\xi$ and $\psi$ are any real values which fall within $[0 ; 1]$.

Static case : we assume that the intervals $S$ and $R_{k}$ are constant. When $P_{a}$ executes a transition $\mathrm{Tr}$ and decides to send a message to $\mathrm{PE}_{\mathrm{b}}$, the time ts between $\operatorname{Tr}$ and the transmission of the message falls within a constant interval $\mathrm{S}$. When $\mathrm{PE}_{b}$ receives the message from $\mathrm{PE}_{\mathrm{a}}$, it can execute a transition $\operatorname{Tr}_{k}$, among $n$ possible transitions $(k=1,2, \ldots, n)$, in a time $\operatorname{tr}_{k}$ belonging to a constant interval $R_{k}$. The interval $S=[\theta ; \phi]$ must satisfy the following equations : $\quad \phi=\psi \times \min _{\mathrm{k}=1}$ to $\mathrm{n}\left(\delta_{\mathrm{k}}-\rho_{\mathrm{a}, \mathrm{b}}\right)$

$$
\theta=\sup (U, 0)+(\phi-\sup (U, 0)) \times \xi
$$

with $\mathrm{U}=\max _{\mathrm{k}=1}$ to $\mathrm{n}\left(\phi+\left(\rho_{\mathrm{a}, \mathrm{b}}-\mu_{\mathrm{a}, \mathrm{b}}\right)-\left(\delta_{\mathrm{k}}-\gamma_{\mathrm{k}}\right)\right)$

Afterwards, we choose the less restrictive solutions for $R_{k}=\left[\tau_{k} ; \omega_{k}\right]$ :

$$
\begin{aligned}
& \text { for } \mathrm{k}=1,2, \ldots, \mathrm{n}: \quad \omega_{\mathrm{k}}=\delta_{\mathrm{k}}-\rho_{\mathrm{a}, \mathrm{b}}-\phi \\
& \tau_{\mathrm{k}}=\sup \left(\gamma_{\mathrm{k}}-\mu_{\mathrm{a}, \mathrm{b}}-\theta ; 0\right)
\end{aligned}
$$

Intuitively, modifying $\psi$ and $\xi$ allows to "move" some timing constraints between two communicating entities.

First dynamic case : we assume that $\mathrm{PE}_{\mathrm{a}}$ sends to $\mathrm{PE}_{\mathrm{b}}$ a message containing ts (Fig. 8.a), and $\mathrm{PE}_{\mathrm{b}}$ calculates dynamically $\mathrm{R}_{\mathrm{k}}$ as a function of ts when it receives the message from $P E_{a}$. The interval $S=[\theta ; \phi]$ must satisfy the following equations : $\quad \phi=\psi \times \min _{\mathrm{k}=1}$ to $\mathrm{n}\left(\delta_{\mathrm{k}}-\rho_{\mathrm{a}, \mathrm{b}}\right)$

$$
\theta=\phi \times \xi
$$

The interval $R_{k}(t s)$ is computed as follows. If ts, which belongs to $[\theta ; \phi]$, is the delay when the message is sent after the execution of $\operatorname{Tr}_{p}$, the receiving entity knows it and can choose :

$$
\begin{array}{ll}
\text { for } \mathrm{k}=1,2, \ldots, \mathrm{n}: & \omega_{\mathrm{k}}(\mathrm{ts})=\delta_{\mathrm{k}}-\rho_{\mathrm{a}, \mathrm{b}}-\mathrm{ts} \\
& \tau_{\mathrm{k}}(\mathrm{ts})=\sup \left(\gamma_{\mathrm{k}}-\mu_{\mathrm{a}, \mathrm{b}}-\mathrm{ts} ; 0\right)
\end{array}
$$

Intuitively, with the information ts, the receiving entity $\mathrm{PE}_{\mathrm{b}}$ can use the time allocated to it to provide the service more efficiently than in the static case. Let us, for instance, assume that some optional tasks are achieved by $\mathrm{PE}_{\mathrm{b}}$ in order to provide a better quality of service, only if $\mathrm{PE}_{\mathrm{b}}$ has enough time. In the static case, $\mathrm{PE}_{\mathrm{b}}$ may estimate that it has not enough time to execute its optional tasks, while in the dynamic case optional tasks will be executed.

Second dynamic case : in comparison with the first dynamic case, we assume that the medium modifies ts into the more accurate information $\mathrm{ts}+\mathrm{tm}$. In this case, $\mathrm{PE}_{\mathrm{b}}$ receives the message with information $\mathrm{ts}+\mathrm{tm}$, and it calculates dynamically the interval $R_{k}$, as a function of ts+tm. $S=[\theta ; \phi]$ is resolved as in the previous case; $\omega_{k}$ and $\tau_{k}$ are calculated by $P E_{b}$ as follows :

$$
\text { for } \mathrm{k}=1,2, \ldots, \mathrm{n}: \quad \begin{aligned}
& \omega_{\mathrm{k}}(\mathrm{ts}+\mathrm{tm})=\delta_{\mathrm{k}}-(\mathrm{ts}+\mathrm{tm}) \\
& \tau_{\mathrm{k}}(\mathrm{ts}+\mathrm{tm})=\sup \left(\eta_{\mathrm{k}}-(\mathrm{ts}+\mathrm{tm}) ; 0\right)
\end{aligned}
$$


Intuitively, with the information $t s+\mathrm{tm}$ the receiving entity $\mathrm{PE}_{\mathrm{b}}$ knows that $\operatorname{Tr}$ has been executed ts+tm before the reception of the message, which is a more accurate information than in the first dynamic case. Due to this fact, in the second dynamic case $\mathrm{PE}_{\mathrm{b}}$ can use the time allocated to it to provide the service more efficiently than in the first dynamic case.

We note that ts and ts+tm, which are transmitted in the dynamic cases, are a relative temporal information. This implies that a global clock is not necessary. We also note that the temporal requirements of the protocol obtained using the approach in [8] are more restrictive than those derived by our improved approach.

Derivation Procedure : it consists of three steps. Step 1, which generates a specification GPST, is similar to step 1 of the non-real-time case.

Step 2 : The aim of this step is : (a) to compute and insert into GPST the static temporal constraints and, in the dynamic cases, some constant parameters which allow to compute the dynamic temporal constraints; (b) to insert ts and tm into the exchanged messages. Therefore, for every structure represented in Fig. 7 and contained in GPST, the following three substeps are performed to transform GPST into GST.

Step 2.1. We compute the interval $S=[\theta ; \phi]$ and:

* Intervals $\mathrm{R}_{\mathrm{k}}, \mathrm{k}=1, \ldots, \mathrm{n}$, in the static case;

* Intervals $\mathrm{X}_{\mathrm{k}}=\mathrm{I}_{\mathrm{k}}-\mathrm{M}_{\mathrm{a}, \mathrm{b}}, \mathrm{k}=1, \ldots, \mathrm{n}$, in the first dynamic case;

Step 2.2 the transition preceding $\mathrm{s}_{\mathrm{a}}^{\mathrm{b}}(\mathrm{p})$ )

- In the static case :

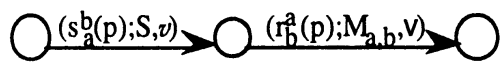

- In the first dynamic case :

- In the second dynamic case :
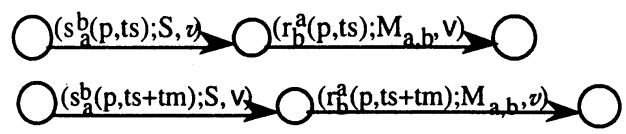

Informally: - the delay between $\operatorname{Tr}$ and $\mathrm{s}_{\mathrm{a}}^{\mathrm{b}}(*)$ falls within $\mathrm{S}=[\theta ; \phi]$;

- the delay between $s_{a}^{b}(*)$ and $r_{b}^{a}(*)$ falls within $M_{a, b}$.

Step 2.3 For each $\mathrm{k}=1, \ldots, \mathrm{n}$, the $t$ th interval $\mathrm{I}_{\mathrm{k}}$ of $c_{\mathrm{k}}$ is replaced by the interval: (i) $\mathrm{R}_{\mathrm{k}}$ in the static case; (ii) $\mathrm{X}_{\mathrm{k}}=\mathrm{I}_{\mathrm{k}}-\mathrm{M}_{\mathrm{a}, \mathrm{b}}$ in the first dynamic case; (iii) $I_{k}$ (i.e., it is not replaced) in the second dynamic case. We note that the GST obtained at Step 2.3 is defined by constant intervals. In dynamic cases, some of these constant intervals do not directly represent timing constraints, but they are used for a dynamic calculation of the time requirements. In fact, for each $k=1, \ldots, n$, the delay between occurrences of $r_{b}^{a}(p)$ and $\operatorname{Tr}_{k}$ must belong to: - the interval $R_{k}$ in the static case;

- an interval $R_{k}(t s)$ which depends on the interval $X_{k}=I_{k}-M_{a, b}$ and on ts;

- an interval $R_{k}(t s+t m)$ which depends on the interval $I_{k}$ and on $t s+t m$. 
Step 3 : This step consists of generating the protocol specification PST $_{i}$ by projecting GST onto the alphabet of events which occur at $S_{i t}, i=1, \ldots, n$. This step is similar to the second step of the non-real-time case, with the difference that intervals $M_{a, b}$ are replaced by $[0 ; \infty]$.

In Sect. 6 and 7, $\psi$ and $\xi$ are taken to be equal to 0.5 , which means that the temporal constraints are equally distributed between the two sites.

\section{SYNTHESIS OF A TIMED X.25 PROTOCOL}

We consider the X.25 service [2]. In order to apply our synthesis method, the $\mathrm{X} .25$ service is made sequential by assuming that the service primitives are ordered and executed sequentially. For that purpose, the following assumptions are made: (i) a new message cannot be sent before the last one is received; and (ii) express data are not supported. The simplified X.25 service obtained will be extended by adding certain temporal requirements between consecutive primitives. In order to give the possibility to both sites to establish a connection, we have used a mechanism of tokens to realize a distributed choice. The following description of this example is based on [4].

Primitives of the simplified X.25 service : Let $U_{1}$ and $U_{2}$ be two users of the network who are located in Site 1 and Site2, respectively. The following service primitives are defined :

- Connection : It may be established between $U_{1}$ and $U_{2}$ if one of them, for instance $\mathrm{U}_{1}$, sends a $\mathrm{CN}$.req to $\mathrm{U}_{2}$. When $\mathrm{U}_{2}$ receives a $\mathrm{CN}$.ind, he may answer either by a DC.req to reject the $\mathrm{CN}$.req, or by a CN.rsp. In the first case $U_{1}$ receives a DC.ind, while in the second case $U_{1}$ receives a $C N . c n f$.

- Disconnection : A disconnection primitive can be used either to reject a CN.req (see above) or to terminate an existing connection. For instance, $\mathrm{U}_{1}$ may send a DC.req and then $\mathrm{U}_{2}$ will receive a DC.ind.

- Data Transfer: Two site linked by a connection may exchange data in both directions. To simplify, we assume that only the party which has initiated the connection can send data. The sending of a message is generated by a DT.req and its reception by a DT.ind.

- Reinitialization : it allows to restore the synchronization between two parties. When a RI.req is generated, for instance by $\mathrm{U}_{1}$, then all the data

being transmitted are removed. The next element to be received by $\mathrm{U}_{2}$ is a RI.ind. $\mathrm{U}_{2}$ answers by a RI.rsp and then $\mathrm{U}_{1}$ will receive a $\mathrm{RI}$.cnf. We assume that the party which requests the reinitialization is the sender of data.

Specification of the Simplified X.25 Service : Our specification contains two blocs $S_{1,2}$ and $S_{2,1}$, where $S_{i, j}$ (see Fig. 9) models the service when $S_{i t e}$ and $S_{i t} j_{j}$ are the sender and the receiver, respectively. The specification of the simplified X.25 is schematized in Fig. 10. The event Token ${ }_{\mathrm{i}}^{\mathrm{j}}$ means that "Site ${ }_{i}$ gives to Site the possibility to establish a connection". We assume that State $1_{1,2}$ is the initial state of the service. 


\section{Temporal constraints added to the simplified X.25 service}

- The delay between $\mathrm{CN}_{\text {.req }} \mathrm{i}$ and $\mathrm{CN}$.ind $\mathrm{j}_{\mathrm{j}}$ belongs to the interval $[1 ; 1.5]$;

- The delay between DC.reqi and DC.indj belongs to the interval $[0.5 ; 1]$;

- The delay between RI.ind $\mathrm{i}_{i}$ and RI.rspi belongs to the interval $[0 ; 0.5]$;

- The delay between DT.req $q_{i}$ and DT.ind ${ }_{j}$ belongs to the interval $[1 ; 1.5]$;

- The delay between DT.rspi and DT.cnf $j_{j}$ belongs to the interval $[1 ; 1.25]$.

Transitions with temporal constraints are represented in grey in Fig. 9 and 10 , with the intervals defining the temporal constraints.

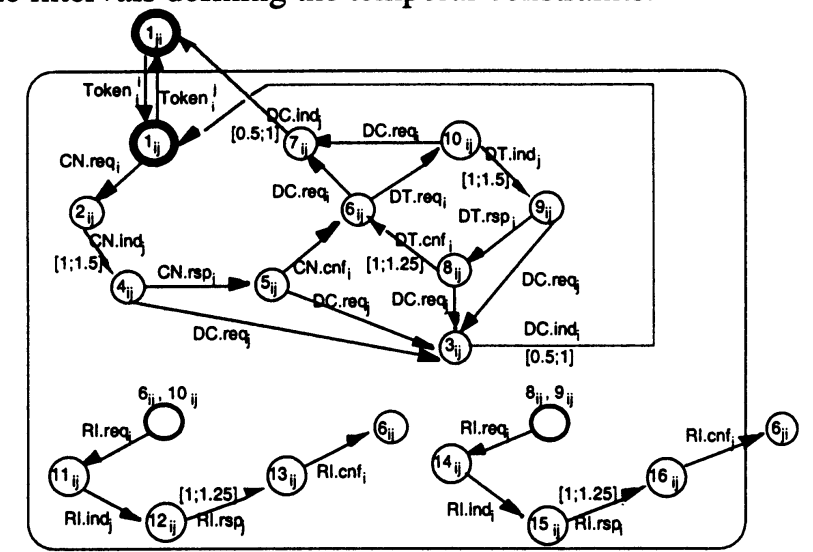

Figure 9. Block $S_{i, j}$

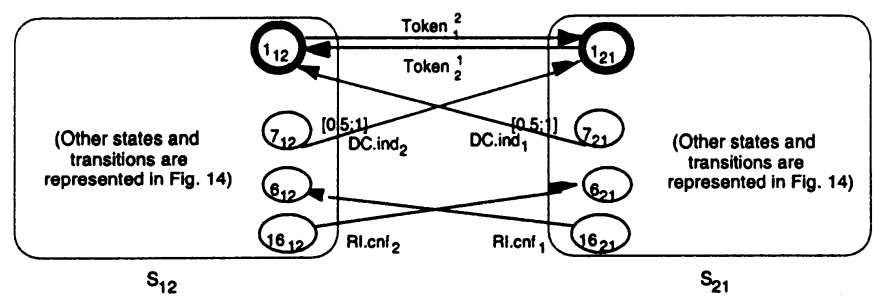

Figure 10. Service specification of the simplified X.25

Protocol Synthesis : The synthesized specifications of the two PEs can be represented by the single $\mathcal{T A}$ of Fig. 11, and where $i$ identifies the PE described and $j$ identifies the other $P E$. Messages sent by each $P E_{i}$ contain the parameters $p_{i}^{k}, k=1, \ldots, 17$, with $p_{i}^{r} \neq p_{i}^{s}$ if $r \neq s$. The initial states of $P E_{1}$ and $P E_{2}$ are identified by 1 and 2 , respectively. To generate these real-time PEs, the following temporal model of the medium has been used: the transit delay of a message falls within $[0.5 ; 0.75]$ when it is transmitted from Site 1 to Site 2 , and within $[0.25 ; 0.5]$ when it is transmitted from Site 2 to Site 1 . To simplify, we give only the results of the static case. The synthesized temporal constraints, which are defined by interval for the transitions represented in grey in Fig. 11, are the following.

Site $_{1}$ : constraint for $s_{1}^{2}\left(p 2_{1}\right), s_{1}^{2}\left(p 3_{1}\right), s_{1}^{2}\left(p 4_{1}\right), s_{1}^{2}\left(p 5_{1}\right), s_{1}^{2}\left(p 10_{1}\right)$ and 
$\mathrm{s}_{1}^{2}\left(\mathrm{p} 11_{1}\right)$ is [0.0625;0.125]; constraint for $\mathrm{s}_{1}^{2}\left(\mathrm{p} 7_{1}\right)$ is [0.25;0.25]; constraint for $s_{1}^{2}\left(p 8_{1}\right)$ and $s_{1}^{2}\left(p 9_{1}\right)$ is [0.25;0.375]; constraint for DT.cnf 1 is [0.375;0.375]; constraint for DT.ind 1 is $[0.375 ; 0.5]$; constraint for DC.ind 1 is [0.125;0.25]; constraint for RI.rsp 1 is [0;0.5].

Site 2 : constraint for $s_{2}^{1}\left(\mathrm{p}_{2}\right), s_{2}^{1}\left(\mathrm{p}_{2}\right), \mathrm{s}_{2}^{1}\left(\mathrm{p} 4_{2}\right), s_{2}^{1}\left(\mathrm{p} 5_{2}\right), s_{2}^{1}\left(\mathrm{p} 10_{2}\right)$ and $s_{2}^{1}\left(\mathrm{p} 11_{2}\right)$ is [0.125;0.25]; constraint for $s_{2}^{1}\left(\mathrm{p}_{2}\right)$ is [0.375;0.375]; constraint for $s_{2}^{1}\left(\mathrm{p}_{2}\right)$ and $s_{2}^{1}\left(\mathrm{pq}_{2}\right)$ is $[0.375 ; 0.5]$; constraint for DT.cnf 2 is [0.25;0.25]; constraint for DT.ind 2 and CN.ind 2 is [0.25;0.375]; constraint for DC.ind 2 is [0;0.125]; constraint for RI.rsp 2 is [0;0.5].

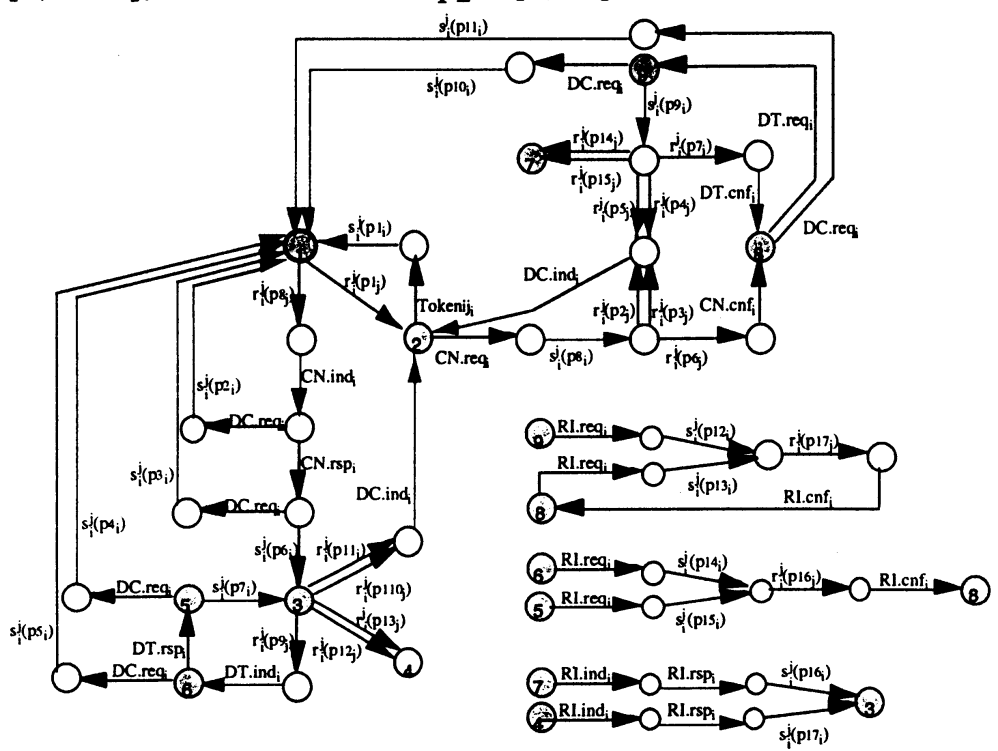

Figure 11. Specification of the synthesized X.25 protocol at Site

\section{EXAMPLE ASSEMBLY SYSTEM}

The following example application of our synthesis method in another area than telecommunications. We consider an assembly system consisting of three robots R1, R2 and R3 and three carpets $\mathrm{C} 1, \mathrm{C} 2$ and $\mathrm{C} 3$. The carpets $\mathrm{C} 1$ and $\mathrm{C} 2$ bring pieces of type $\mathrm{P} 1$ and $\mathrm{P} 2$, respectively, and carpet $\mathrm{C} 3$ takes away the assembled pieces. Robot R1 takes a piece $\mathrm{P} 1$, and puts it on a table $\mathrm{T}$ for the assembly. Robot R2 takes a piece P2 and assembles it with the piece $\mathrm{P} 1$ which is on the table $\mathrm{T}$. Robot $\mathrm{R} 3$ removes the defective pieces. The details of this example are given in [9].

Protocol entities : There are six PEs, R1, R2, R3, C1, C2 and C3, which correspond to the three robots and the three carpets, respectively. Table $\mathrm{T}$ is not considered as an entity since it is passive. A piece is denoted $\mathrm{Pi}$.

Service primitives : They are the following $(i=1,2,3$, and $j=1,2)$ :

MOVE.Carpet $\mathrm{Ci}$ : $\mathrm{Ci}$ is actuated; ARRIVED.Piece $\mathrm{Ci}$ : $\mathrm{Ci}$ has detected that a 
$\mathrm{Pi}$ has reached its destination; STOP.Carpet $\mathrm{Ci}: \mathrm{Ci}$ is stopped ; CHECK.Piece $R j$ : Rj begins to check Pj ; TAKE.PieceRj : Rj takes a Pj from $\mathrm{Cj}$; TAKE.Piece ${ }_{\mathrm{R} 3}: \mathrm{R} 3$ takes a $\mathrm{P} 1$ from $\mathrm{C} 1$; TAKE.Piece2 $\mathrm{R} 3: \mathrm{R} 3$ takes a piece $\mathrm{P} 2$ from $\mathrm{C} 2$; TAKE.AssPieces $\mathrm{R} 3: \mathrm{R} 3$ takes an assembled piece from table T ; PUT.Piece 1 : R1 puts a P1 on T ; PUT.AssPiecesR2 : R2 puts an assembled piece on C3; ASS.PiecesR2 : R2 assembles $\mathrm{P} 1$ and $\mathrm{P} 2$.

Scenario of the Service : From the initial state where the whole system is stopped, the scenario of the service is the following:

Step 1: $\mathrm{Cl}$ is actuated (MOVE.Carpet $\mathrm{Cl}$ )

Step 2: $\mathrm{Pl}$, which is on $\mathrm{Cl}$, reaches its destination (ARRIVED.Piece $\mathrm{C1}$ )

Step $3: \mathrm{C} 1$ is stopped (STOP.Carpet $\mathrm{C1}$ )

Step $4:$ R1 checks P1 (CHECK.PieceR1):

Step 5: If $\mathrm{P} 1$ is bad then $\mathrm{R} 3$ takes it off (TAKE.Piece ${ }_{\mathrm{R} 3}$ ), and goto Step 1 .

Step 6: If $\mathrm{P} 1$ is good then $\mathrm{R} 1$ takes it from $\mathrm{Cl}$ (TAKE.Piece $\mathrm{R} 1$ ) and

Step 7: $\mathrm{R} 1$ puts $\mathrm{Cl}$ on the table $\mathrm{T}$ (PUT.Piece $\mathrm{R} 1$ )

Step $8: \mathrm{C} 2$ is actuated (MOVE.Carpet 2 )

Step 9: $\mathrm{P2}$, which is on $\mathrm{C}$, reaches its destination (ARRIVED.Piece $\mathrm{C} 2$ )

Step 10: Carpet C2 is stopped (STOP.CarpetC2)

Step 11: Robot R2 checks P2 (CHECK.PieceR2):

Step 12 : If $\mathrm{P} 2$ is bad then $\mathrm{R} 3$ takes it off (TAKE.Piece2R3) and goto Step 8.

Step 13: If P2 is good then R2 takes it from C2 (TAKE.PieceR2) and

Step $14: \mathrm{R} 2$ assembles $\mathrm{P} 2$ with P1 on the table T (ASS.PiecesR2).

Step 15 : If the assembly is bad, which it is detected by R2, then R3 takes it off

(TAKE.AssPiecesR3), and goto Step 1.

Step 16: If the assembly is good then $\mathrm{R} 2$ takes it and puts it on carpet $\mathrm{C} 3$ (PUT.AssPiecesR2)

Step 17 : Carpet $\mathrm{C} 3$ is actuated (MOVE.Carpet $\mathrm{C} 3$ )

Step 18 : The assembled pieces reach their destination, which is detected by carpet $\mathrm{C} 3$ (ARRIVED.PieceC 3 ).

Step 19 : Carpet C3 is stopped (STOP.Carpet $\mathrm{C} 3$ ), and goto Step 1.

Temporal constraints added to the service

- Between MOVE.Carpet $\mathrm{Ci}$ and ARRIVED.Piece $\mathrm{Ci}_{\mathrm{i}}$ : $[10 ; 20] ;(\mathrm{i}=1,2,3)$

- Between ARRIVED.Piece $\mathrm{Ci}_{\mathrm{i}}$ and STOP.Carpet $\mathrm{Ci}$ : $[0.5 ; 2] ;(\mathrm{i}=1,2,3)$

- Between STOP.CarpetCi and CHECK.Piece $\mathrm{Ri}:[5 ; 8] ;(\mathrm{i}=1,2)$

- Between CHECK.PieceRi and TAKE.PieceiR3 : $[5 ; 8] ;(i=1,2)$

- Between ASS.Piecer2 and TAKE.AssPieces 3 : [5;8];

- Between CHECK.Piece Ri and TAKE.Piece ${ }_{R i}:[1 ; 2] ;(i=1,2)$

- Between TAKE.Piece $R_{R}$ and PUT.Piece $R 1:[1 ; 2]$;

- Between TAKE.PieceR2 and ASS.PiecesR2 : [4;10];

- Between PUT.PieceR1 (or TAKE.Piece2R3) and MOVE.CarpetC2 : [5;10];

- Between PUT.AssPiecesR2 and MOVE.Carpet $33:[5 ; 10]$;

- Between ASS.PiecesR2 and PUT.AssPiecesR2: [2;5]; 
- Between TAKE.Piece $1_{R 3}$ (or TAKE.AssPiecesR3 or STOP.CarpetC3) and MOVE.CarpetCl : [6;10]; $(\mathrm{i}=1,2)$

The service specification is represented in Fig. 12, where transition $\operatorname{Tr}_{1}$ corresponds to Step $\mathrm{i}$ of the scenario. The temporal constraint of each transition is defined by a single interval (which is shown in Fig. 12).

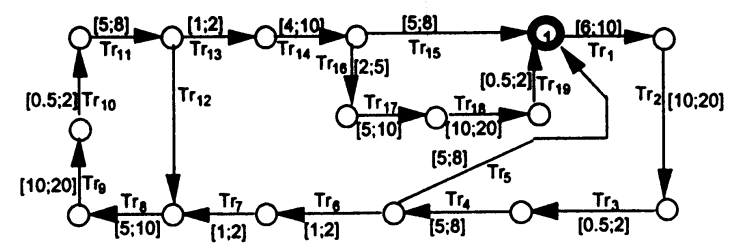

Figure 12. Service specification of the assembling system

Protocol Synthesis : The specifications synthesized in the static case are represented in Fig. 13, and consist of six $\mathcal{T} \mathcal{A}$ s modeling the the three robots and the three carpets, respectively. To generate these real-time PEs, the transit delay of all messages has been assumed to belong to [2;5].

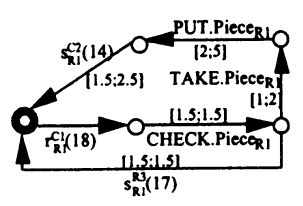

(a) Robot R1

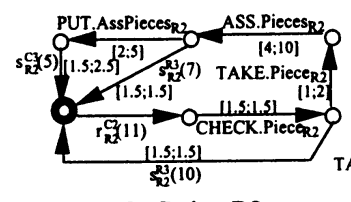

(b) Robot R2

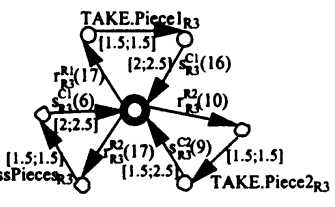

(c) Robot R3

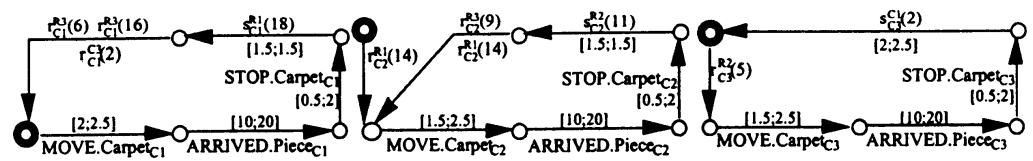

(d) Carpet $\mathrm{Cl}$

(e) Carpet $\mathrm{C} 2$

(f) Carpet $\mathrm{C} 3$

Figure 13. Synthesized specifications of the robots and the carpets in the static case

\section{CONCLUSION}

A method for deriving real-time protocols [8] is improved and extended in the present paper. Our method imposes requires that the service is sequential. We are presently investigating an approach using the following three steps : (a) the given service specification $S$ is transformed into a sequential service, called $S^{s e q}$; (b) a protocol $P^{s e q}$ providing the sequential service $S^{s e q}$ is synthesized by using our method; the obtained protocol is a kind of "skeleton" of a protocol $P$ providing $S$; (c) $P^{s e q}$ is transformed in order to obtain a protocol $P$ which provides $S$.

We note that Step (b) is realized automatically by the method presented in the present paper, and that Step (a) has been applied manually in the examples of Sect. 6 and 7 to make our method applicable. We therefore try to find a systematic way to achieve steps (a) and (c). 


\section{References}

[1] R. ALur and D.Dill, "Automata for Modeling Real-Time Systems," in Proceedings of the 17th Intern. Coll. on Automata, Languages and Programming, Lecture Notes in Comp. Sci. 443, Ed. Springer-Verlag, Warwick, UK, 1990.

[2] R.J. Deasington, "Protocoles X.25 pour les réseaux à commutation de paquets," Masson, 1987.

[3] R. Gotzhein and G. v. Bochmann, "Deriving Protocol Specifications from Service Specifications Including parameters," ACM Transactions on Computer Systems, Vol. 8, N 4, pp. 255-283, 1990.

[4] Y. Iraqi, "Synthèse du protocole X.25 simplifié avec contraintes de temps. Utilisation de l'outil de Khoumsi," Report of a project realized at the University of Montreal, April, 1996.

[5] C. Kant, T. Higashino and G.v. Bochmann, "Deriving Protocol Specifications from Service Specifications Written in LOTOS," Distributed Computing, Vol. 10, № 1, pp. 29-47, 1996.

[6] M. Kapus Kolar, "Deriving protocol specifications from service specifications with heterogeneous timing requirements." in Proceedings of the IEEE Int. Conf. on Software Engineering for real time systems, United-Kingdom, 1991.

[7] M. Kapus Kolar and J. Rugelj, "Deriving protocol specifications from service specifications with simple relative timing requirements," in Proceedings of ISMM Int. Workshop on parallel computing, Italy, 1991.

[8] A. Khoumsi, G.v. Bochmann, and R. Dssouli, "Dérivation de spécifications de protocole à partir de spécifications de service avec des contraintes temps-réel," Revue Réseaux et informatique répartie (RIR), Vol.4, N 1 , April 1994.

[9] E. Madja, "Dérivation de protocoles pour applications temps réeel. Application au système d'assemblage," Report of a project realized at the University of Montreal, April, 1996.

[10] K.Saleh and R. Probert, "A service-based method for the synthesis of Communications protocols," International Journal of Mini and Microcomputers, Vol. 12, N 3, pages 97-103, December 1990.

[11] H. Yamaguchi, K. Okano, T. Higashino and K. Taniguchi, "Synthesis of protocol entities specifications from service specifications in a Petri Net model with registers,", in Proceedings of IEEE Parallel and Distributed Computing Systems, 1995. 